\title{
The Origins Space Telescope: mission concept overview
}

\section{Leisawitz, E. Amatucci, R. Carter, M. DiPirro, A. Flores, et al.}

D. Leisawitz, E. Amatucci, R. Carter, M. DiPirro, A. Flores, J. Staguhn, C. Wu, L. Allen, J. Arenberg, L. Armus, C. Battersby, J. Bauer, R. Bell, P. Beltran, D. Benford, E. Bergin, C. M. Bradford, D. Bradley, D. Burgarella, S. Carey, D. Chi, A. Cooray, J. Corsetti, E. De Beck, K. Denis, L. Dewell, M. East, S. Edgington, K. Ennico, L. Fantano, G. Feller, D. Folta, J. Fortney, J. Generie, M. Gerin, Z. Granger, G. Harpole, K. Harvey, F. Helmich, L. Hilliard, J. Howard, M. Jacoby, A. Jamil, T. Kataria, S. Knight, P. Knollenberg, P. Lightsey, S. Lipscy, E. Mamajek, G. Martins, M. Meixner, G. Melnick, S. Milam, T. Mooney, S. H. Moseley, D. Narayanan, S. Neff, T. Nguyen, A. Nordt, J. Olson, D. Padgett, M. Petach, S. Petro, J. Pohner, K. Pontoppidan, A. Pope, D. Ramspacher, T. Roellig, I. Sakon, C. Sandin, K. Sandstrom, D. Scott, K. Sheth, J. Steeves, K. Stevenson, L. Stokowski, E. Stoneking, K. Su, K. Tajdaran, S. Tompkins, J. Vieira, C. Webster, M. Wiedner, E. L. Wright, J. Zmuidzinas, "The Origins Space Telescope: mission concept overview," Proc. SPIE 10698, Space Telescopes and Instrumentation 2018: Optical, Infrared, and Millimeter Wave, 1069815 (24 July 2018); doi: 10.1117/12.2313823

Event: SPIE Astronomical Telescopes + Instrumentation, 2018, Austin, Texas, United States 


\section{The Origins Space telescope: mission concept overview}

D. Leisawitz ${ }^{\mathrm{a}}$, E. Amatucci ${ }^{\mathrm{a}}$, R. Carter ${ }^{\mathrm{a}}$, M. DiPirro ${ }^{\mathrm{a}}$, A. Flores ${ }^{\mathrm{a}}$, J. Staguhn ${ }^{\mathrm{a}, \mathrm{b}}$, C.Wu ${ }^{\mathrm{a}}$, L. Allen ${ }^{\mathrm{c}}, \mathrm{J}$. Arenberg $^{\mathrm{d}}$, L. Armus ${ }^{\mathrm{e}}$, C. Battersby ${ }^{\mathrm{f}}$, J. Bauer ${ }^{\mathrm{g}}$, R. Bell ${ }^{\mathrm{h}}$, P. Beltran ${ }^{\mathrm{a}}$, D. Benford ${ }^{\mathrm{i}}$, E. Bergin', C.M. Bradford $^{\mathrm{k}}$, D. Bradley ${ }^{\mathrm{a}}$, D. Burgarella ${ }^{\mathrm{l}}$, S. Carey ${ }^{\mathrm{e}}$, D. Chi ${ }^{\mathrm{d}}$, A. Cooray ${ }^{\mathrm{m}}$, J. Corsettia ${ }^{\mathrm{a}}$, E. De Beck ${ }^{\mathrm{n}}$, K.

Denis $^{\text {a }}$, L. Dewell , M. East ${ }^{\mathrm{c}}$, S. Edgington ${ }^{\mathrm{h}}$, K. Ennico ${ }^{\mathrm{o}}$, L. Fantano, G. Feller ${ }^{\mathrm{h}}$, D. Folta ${ }^{\mathrm{a}}$, J.

Fortney $^{\mathrm{p}}$, J. Generie ${ }^{\mathrm{a}}$, M. Gerin ${ }^{\mathrm{q}}$, Z. Granger ${ }^{\mathrm{h}}$, G. Harpole ${ }^{\mathrm{d}}$, K. Harvey ${ }^{\mathrm{c}}$, F. Helmich ${ }^{\mathrm{r}}$, L. Hilliard ${ }^{\mathrm{a}}$, J. Howard ${ }^{\mathrm{a}}$, M. Jacoby ${ }^{\mathrm{h}}$, A. Jamil', T. Kataria ${ }^{\mathrm{k}}$, S. Knight ${ }^{\mathrm{s}}$, P. Knollenberg ${ }^{\mathrm{d}}$, P. Lightsey , S. Lipscy ${ }^{\mathrm{s}}$, E.

Mamajek $^{k}$, G. Martins ${ }^{\mathrm{a}}$, M. Meixner, ${ }^{\mathrm{b}, \mathrm{t}}$, G. Melnick ${ }^{\mathrm{u}}$, S. Milam ${ }^{\mathrm{a}}$, T. Mooney ${ }^{\mathrm{c}}$, S.H. Moseley ${ }^{\mathrm{a}}$, D.

Narayanan $^{\mathrm{v}}$, S. Neff ${ }^{\mathrm{a}}, \mathrm{T}$. Nguyen ${ }^{\mathrm{d}}$, A. Nordt ${ }^{\mathrm{h}}$, J. Olson ${ }^{\mathrm{h}}$, D. Padgett ${ }^{\mathrm{k}}$, M. Petach ${ }^{\mathrm{d}}$, S. Petro ${ }^{\mathrm{a}}$, J.

Pohner $^{\mathrm{d}}$, K. Pontoppidan', A. Pope ${ }^{\mathrm{w}}$, D. Ramspacher, ${ }^{\mathrm{a}}$, T. Roellig ${ }^{\mathrm{b}}$, I. Sakon ${ }^{\mathrm{x}}$, C. Sandina ${ }^{\mathrm{a}}$ K.

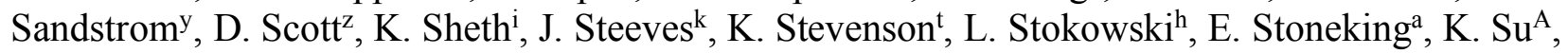

K. Tajdaran ${ }^{\mathrm{h}}$, S. Tompkins ${ }^{\mathrm{a}}$, J. Vieira ${ }^{\mathrm{B}}$, C. Webster ${ }^{\mathrm{a}}$, M. Wiedner ${ }^{\mathrm{q}}$, E.L. Wright ${ }^{\mathrm{C}}$, and J. Zmuidzinas ${ }^{\mathrm{D}}$

a NASA Goddard Space Flight Center, 8800 Greenbelt Rd., Greenbelt, MD 20771 USA

${ }^{\mathrm{b}}$ Johns Hopkins University, Baltimore, MD, USA

${ }^{c}$ Harris Corporation, Melbourne, FL, USA

${ }^{\mathrm{d}}$ Northrop-Grumman Aerospace Systems, Redondo Beach, CA, USA

${ }^{\mathrm{e}}$ Caltech/Infrared Processing and Analysis Center, Pasadena, CA, USA

${ }^{\mathrm{f}}$ Department of Physics, University of Connecticutt, Storrs, CT, USA

g Astronomy Department, University of Maryland, College Park, MD, USA

${ }^{\mathrm{h}}$ Lockheed-Martin Advanced Technology Center, Palo Alto, CA, USA

${ }^{i}$ Astrophysics Division, Science Mission Directorate, NASA Headquarters, Washington, DC, USA

j Department of Astronomy, University of Michigan, Ann Arbor, MI, USA

${ }^{\mathrm{k}}$ Caltech/Jet Propulsion Laboratory, Pasadena, CA, USA

${ }^{1}$ Laboratoire d'Astrophysique de Marseille, Marseille, France

${ }^{m}$ Department of Physics and Astronomy, University of California, Irvine, Irvine, CA, USA

${ }^{n}$ Dept. of Space, Earth and Environment, Chalmers Institute of Technology, Gothenburg, Sweden

${ }^{\circ}$ NASA Ames Research Center, Mountain View, CA, USA

${ }^{\mathrm{p}}$ Astronomy and Astrophysics Dept., University of California, Santa Cruz, Santa Cruz, CA, USA

q Sorbonne Université, Observatoire de Paris, CNRS, LERMA, F-75014, Paris, France

${ }^{\mathrm{r}}$ Netherlands Institute for Space Research (SRON), Groningen, The Netherlands

${ }^{\mathrm{s}}$ Ball Aerospace Corporation, Boulder, CO, USA

${ }^{\mathrm{t}}$ Space Telescope Science Institute, Baltimore, MD, USA

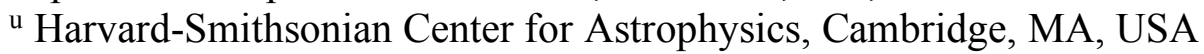

v Department of Astronomy, University of Florida, Gainesville, FL, USA

${ }^{w}$ Department of Astronomy, University of Massachusetts, Amherst, MA, USA

${ }^{x}$ School of Science, The University of Tokyo, 7-3-1 Hongo, Bunkyo-ku, Tokyo, Japan

${ }^{y}$ CASS, University of California, San Diego, San Diego, CA, USA

${ }^{z}$ Dept. of Physics and Astronomy, The University of British Columbia, Vancouver, BC, Canada

A Dept. of Astronomy and Steward Observatory, The University of Arizona, Tucson, AZ, USA

B Department of Astronomy, University of Illinois, Urbana-Champaign, Urbana, IL, USA

${ }^{\mathrm{C}}$ Division of Astronomy and Astrophysics, UCLA, Los Angeles, CA, USA

D Division of Physics, Mathematics and Astronomy, Caltech, Pasadena, CA, USA 


\begin{abstract}
The Origins Space Telescope (OST) will trace the history of our origins from the time dust and heavy elements permanently altered the cosmic landscape to present-day life. How did the universe evolve in response to its changing ingredients? How common are life-bearing planets? To accomplish its scientific objectives, OST will operate at mid- and far-infrared wavelengths and offer superlative sensitivity and new spectroscopic capabilities.

The OST study team will present a scientifically compelling, executable mission concept to the 2020 Decadal Survey in Astrophysics. To understand the concept solution space, our team studied two alternative mission concepts. We report on the study approach and describe both of these concepts, give the rationale for major design decisions, and briefly describe the mission-enabling technology.
\end{abstract}

\title{
1. INTRODUCTION
}

In astrophysics, the far-infrared - wavelengths from about 30 to $600 \mu \mathrm{m}$ - is arguably the most information rich, underexploited region of the electromagnetic spectrum. Past far-IR space missions, including Herschel ${ }^{1}$, the largest space telescope flown to date, operated orders of magnitude away from fundamental sensitivity limits and offered diffractionlimited angular resolution comparable to that of the telescopes used by Galileo four centuries ago. Yet with these past missions, including IRAS ${ }^{2}, \mathrm{COBE}^{3}, \mathrm{ISO}^{4}, \mathrm{Spitzer}^{5}$, and $\mathrm{AKARI}^{6}$, as well as Herschel, the astrophysics community has made tremendous scientific strides and surprising discoveries, gaining insight into the process of star formation, the composition and energetics of the interstellar medium, and precisely characterizing the very early universe.

There is much more to be done. For example, we have not yet learned how the conditions for habitability sometimes arise during the planet formation process. In particular, how does water make its way from the interstellar medium to a planet just warm enough to melt it, but not so hot as to evaporate it. Also, for example, we have only barely begun to characterize the physical and chemical conditions in nascent galaxies to understand how galaxies changed throughout cosmic history. Interstellar dust formed early, and when it did the process of star formation was forever altered. The dust absorbs and blocks UV/visible starlight and emits in the far-IR. A rich spectrum of line emission from water vapor, the dominant coolants of the interstellar medium, and important diagnostics of the hardness of the interstellar radiation field are found in the far-IR, as are broad-band spectral features of frozen water. To access these diagnostics, the astrophysics community needs a far-IR space telescope that approaches natural background sensitivity limits.

A NASA-sponsored, community-led study of the Origins Space Telescope (OST) is underway. This study will culminate in a report to the US National Academies when it conducts the 2020 Decadal Survey in Astrophysics. The OST Science and Technology Definition Team (STDT) prioritized scientific objectives attainable with a telescope that provides superlative sensitivity but does not require a large improvement in angular resolution relative to the $3.5 \mathrm{~m}$ Herschel Space Observatory. Thus, the STDT reached an early decision that OST would be a single-aperture telescope.

The scope of this paper is limited to a summary description of the OST mission concept, while parallel papers describe different facets of the mission study. Meixner et al. $(2018)^{7}$ present the scientific motivation for OST. DiPirro et al. $(2018)^{8}$ describe the cryo-thermal system design and the attainability of the $4 \mathrm{~K}$ optical system operating temperature requirement with current state-of-the-art cryocooler technology. Knight et al. (2018) ${ }^{9}$ present the OST wavefront error budget, and Lightsey et al. (2018) ${ }^{10}$ present preliminary results of a stray light analysis. Matsuo et al. $(2018)^{11}$ describe a pupil densification technique that can be used to enhance stability and enable extremely precise spectroscopic measurements of transiting exoplanets to search for planets with biosignatures in the mid-IR $(5-25 \mu \mathrm{m})$. The four OST instruments are described in papers by Bradford et al. (2018) ${ }^{12}$, Sakon et al. $(2018)^{13}$, Wiedner et al. $(2018)^{14}$, and Staguhn et al. (2018) ${ }^{15}$. This paper presents two concepts for OST in which the study team has invested most of its effort, while Arenberg et al. (2018) ${ }^{16}$ present a third alternative concept ("cold JWST") based on the possibility that great cost savings would result if existing JWST designs, supporting hardware, and test facilities were reused, with minimal modification, to make OST. Papers on detector technology relevant to OST can be found in the Proceedings volume "Millimeter, Submillimeter, and Far-Infrared Detectors and Instrumentation for Astronomy IX" (Conference 10708).

This paper is organized as follows. Section 2 provides contextual information and describes the study approach adopted by the OST STDT. Section 3 presents and contrasts the two main mission design concepts. The OST instruments are briefly described in Section 4, and enabling technology is briefly described in Section 5. We summarize in Section 6. 


\section{STUDY APPROACH}

Our concept for the Origins Space Telescope is of the community, by the community, and for the community. In the NASA Astrophysics Roadmap, Enduring Quests, Daring Visions $(2013)^{17}$, a committee representing the astrophysics community envisioned a "Far-IR Surveyor" mission that would offer measurement capabilities vastly superior to any prior far-IR mission. After further community vetting, NASA decided to sponsor studies of OST and three other large missions in response to Roadmap or past Decadal Survey goals. The OST study is "by the community" because the STDT actively engages community members in developing and documenting potential science objectives for the mission. Finally, if OST is endorsed by the 2020 Decadal Survey and developed by NASA, OST Guest Observers will use it to answer the mission-driving science questions and undoubtedly make unexpected, transformative discoveries.

The top-level goal of the OST study is to deliver a scientifically compelling, executable mission concept to the 2020 Astrophysics Decadal Survey by June 2019. While the engineering design is still developing, it is responsive to the STDT's prioritized scientific goals and fast approaching executability. NASA has a very strong track record when it comes to implementing the large missions recommended in past Decadal Surveys.

The OST study team adopted an iterative approach to mission concept development (Figure 1). This approach enables an exploration of the mission design solution space. The approach works best when a cost target is established and iteration proceeds through three or more design cycles ${ }^{18}$, affording an opportunity to bracket the target with the first two cycles, and then interpolate in an effort to maximize science return at a price point. The resources available for this study are sufficient to cycle through only two iterations. Since a large Decadal mission is not strictly cost-capped, the STDT decided first to explore a concept with no cost constraint. This enabled us to set an upper bound on the solution space, driven by ambitious and wide-ranging science objectives. We refer to the result as OST "Concept 1." Based on the NASA budget thought likely to be available for a large mission after WFIRST, the STDT then established a \$5B cost target for "Concept 2," the product of a second design cycle. Knowing that a third cycle would not be achievable in the pre-Decadal time frame, we decided to use flight system mass as a proxy for cost at $\$ 1 \mathrm{M} / \mathrm{kg}$ throughout the engineering design study, and subtracted 30\% from \$5B for margin. A real cost estimate will only be derivable in the end, so the team is paying close attention to scientific priorities, mass, and descope options during the Concept 2 design cycle. Once the design is complete, we will use both parametric and analogy-based methods to estimate cost, basing the former on a detailed Master Equipment List (MEL) for all flight system components, including the instruments. Depending on the estimated cost of Concept 2 relative to the targeted cost, the STDT may decide to present descope or upscope options, with benefits and costs, along with a baseline mission concept to the Decadal Survey.

\section{MISSION CONCEPT SUMMARY}

Concept 1 (Figure 2) was described in the OST Interim Report, which will soon be released to the public, but the STDT is likely to recommend Concept 2, or a derivative of Concept 2, to the Decadal Survey, so we focus on Concept 2 here.

With Concept 1 as an upper bound, it was clear that Concept 2 would have to be "less ambitious"; the telescope would have to be smaller than $9 \mathrm{~m}$. At the same time, the STDT wanted to retain the highest priority science. Thus, priorities were established through blind voting and deliberation. For Concept 2, the motivating science questions and goals established as principal mission design drivers by the $\mathrm{STDT}^{7}$ are as follows:

1. How common are life-bearing planets orbiting M dwarf stars?

$>$ OST will search for biosignatures in the mid-infrared in a sample of star-transiting exoplanets large enough to make a null result interesting.

2. How do the conditions for habitability develop during the process of planet formation?

$>$ OST will follow the trail of water (vapor and ice) from the interstellar medium to nascent planets.

3. How do galaxies form stars, grow their central supermassive black holes, and make heavy elements over time?

$>$ OST will probe the universe deeply in key dust-penetrating diagnostic spectral lines. 


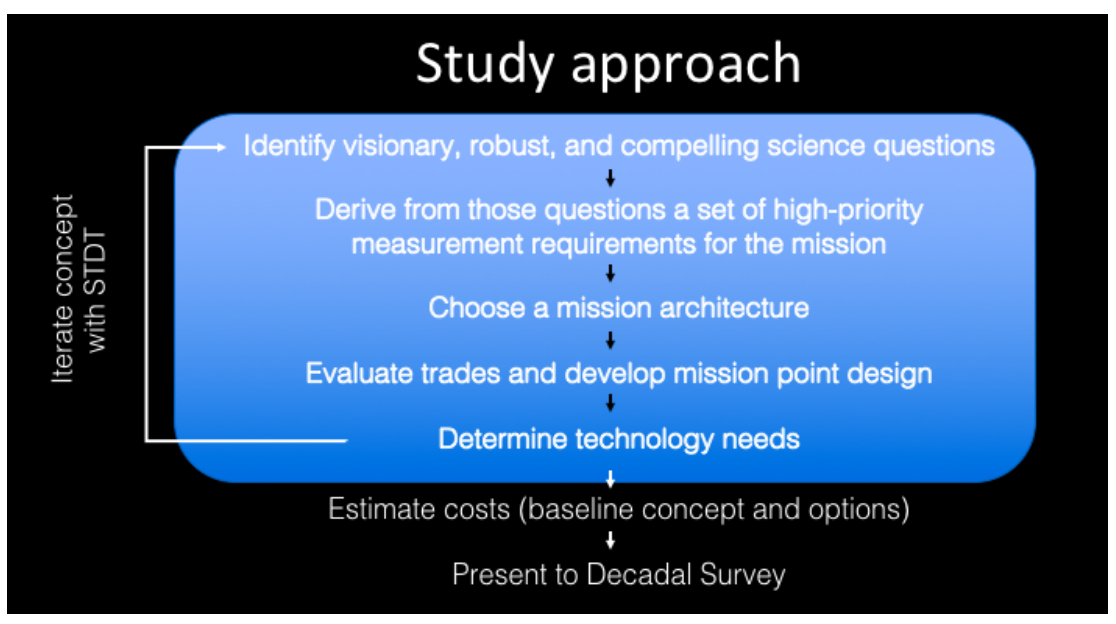

Figure 1. An iterative approach to mission design concept development enables the OST study team to explore solution space in an effort to develop a concept that maximizes science yield with an executable mission at an affordable cost.

Although OST was originally conceived as a far-IR telescope, the STDT recognized the importance of goal 1 and extended the short wavelength cutoff to $5 \mu \mathrm{m}$, enabling searches for the coexistence of methane and ozone in an exoplanet's atmosphere, that combination (or others sustainable only with a biogenic process) constituting a biosignature. ${ }^{19}$

Working with members of the community, the STDT derived quantitative measurement requirements corresponding to these goals. First and foremost, OST will have to provide exquisite sensitivity. Goals 2 and 3 both require $5 \sigma$ sensitivity to $10^{-20} \mathrm{~W} \mathrm{~m}^{-2}$ far-IR spectral lines in 1 hour. Second, OST instruments will have to provide spectroscopy with resolving power $(\lambda / \Delta \lambda)$ ranging from 10 to $>10^{6}$ in approximately order-of-magnitude increments. Third, OST will have to be able to survey large areas in a reasonable amount of observing time. For example, to achieve goal 3, the STDT envisions that OST will be used to conduct a deep extragalactic "Legacy survey" covering $10 \mathrm{deg}^{2}$ in 1000 hours. This survey will become one layer in a "wedding cake" survey comprising shallow, deep, and ultra-deep layers of progressively narrow sky coverage. Fourth, OST will have to provide superlative stability $(<5 \mathrm{ppm})$ in the spectral range 5 to $25 \mu \mathrm{m}$ to enable a fruitful search for biosignatures in the spectra of transiting exoplanets. Note that the prioritized scientific objectives do not require high (sub-arcsecond) angular resolution. A cold (4 K) telescope, diffraction limited at $30 \mu \mathrm{m}$ and equipped with next-generation detectors in high pixel count arrays can approach the astronomical background photon noise limit and satisfy the OST requirements.

During a brainstorming workshop with mission-experienced STDT members and industry partners Ball Aerospace, Northrop-Grumman Aerospace Systems, Lockheed-Martin, Harris Corporation, and Raytheon, a variety of architecture options were proposed and evaluated before the study team chose an architecture for Concept 2. Science return, complexity and heritage were important metrics. The STDT's exoplanet science working group concluded that a telescope smaller than $5 \mathrm{~m}$ would yield too few biosignature measurements to satisfy a critical objective. Noting that planned large launch vehicles could accommodate a $5 \mathrm{~m}$ telescope without folding and deployment, the study team traded complexity vs. risk. The telescope could be folded and packaged for launch in an existing fairing (more complex) or not folded and launched in a fairing that doesn't yet exist (greater risk). Considering that three potentially usable launch vehicles are presently under study or development - NASA's SLS, as shown in Figure 1; the Space X BFR; and the Blue Origins New Glenn, and after consultation with representatives of The Aerospace Corporation, the team decided that the benefits of greatly reduced complexity outweighed the unlikely possibility that no suitable launch vehicle would exist by the mid-2030s, when OST would fly. The study team adopted a Spitzer-like architecture for Concept 2 (Figure 3) that would provide the same light collecting area as JWST $\left(25 \mathrm{~m}^{2}\right)$ and have no complex deployments. 

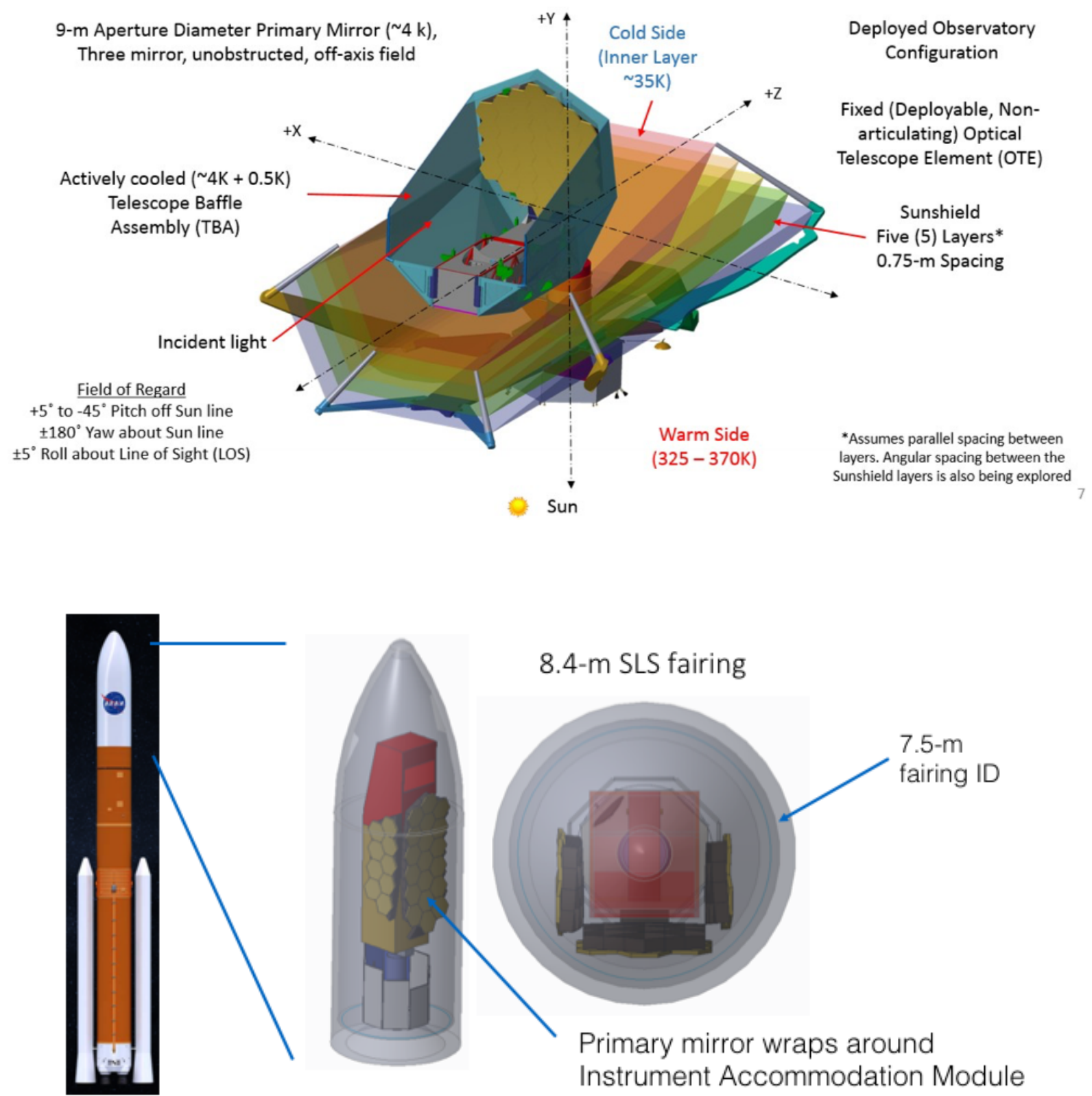

Figure 2. Concept 1 has an open architecture and resembles JWST with its large multi-layer, deployable sunshield. The primary mirror is unobstructed, segmented, and hexagonal, measuring $9.1 \mathrm{~m}$ from tip to tip. The Instrument Accommodation Module houses 5 science instruments. The flight system fits into a NASA Space Launch System (SLS) $8.4 \mathrm{~m}$ fairing, but requires an elaborate deployment sequence to reach its operational configuration. Concept 1 satisfies essentially all scientific objectives considered interesting for the mission. 


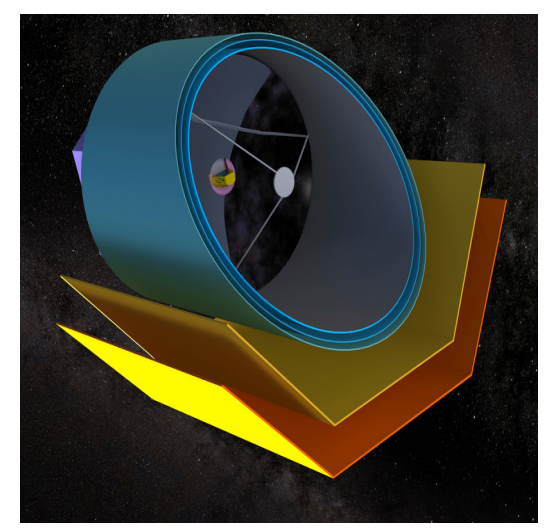

Figure 3. OST Concept 2 has a Spitzer-like architecture (telescope in a "barrel" protected by sunshades) with a cryocooled $4 \mathrm{~K}$ telescope (no expendable cryogen).

Key attributes of OST mission Concepts 1 and 2 are compared in Table 1. In both cases, the telescope is diffraction limited at $30 \mu \mathrm{m}$, and the mid-IR instrument uses a deformable mirror to make mid-spatial frequency wavefront corrections and extend diffraction-limited performance to $5 \mu \mathrm{m}$. In both cases, the telescope is a three-mirror anastigmat, as such a configuration provides a larger usable field of view, improved imaging performance, and enables incorporation of a Field Steering Mirror. The Concept 1 telescope is unobstructed (off-axis pupil), whereas the Concept 2 telescope is on-axis. Like other important parameters, the on-axis configuration was chosen after a trade study because it is easier to package and has more symmetric segments, making it easier to fabricate and test. The Concept 2 telescope (Figure 4 ) has a circular pupil and thus provides cleaner, symmetric point spread functions. The most important differences between the two concepts are in overall complexity, telescope size, and number of instruments: five instruments in Concept 1 , four in Concept 2 .

Like JWST, the primary mirror is comprised of segments, but they are pie wedges rather than hexagons, as shown in Figure 4. The OST primary mirror has inner and outer rings of segments, and all segments in each ring have identical optical prescriptions. This will reduce design, manufacturing, validation, and verification time, and keep the number of required flight spare units to a minimum. With $30 \mu \mathrm{m}$ diffraction-limited performance, the OST telescope's wavefront error budget is relaxed by an order of magnitude compared to JWST. The OST wavefront error budget ${ }^{9}$ indicates that 3dof segment position control - allowing adjustment of tip, tilt, and piston - is sufficient to meet performance requirements. For comparison, the JWST primary mirror segments are adjustable in seven degrees of freedom, including radius-of-curvature. The segment actuators can borrow JWST heritage. Whereas "cryo-null figuring" was critical to the JWST mirror segment development program, the OST mirror segments can bypass this step, as mirrors that will satisfy OST's relaxed surface figure error requirement at $4 \mathrm{~K}$ can be directly manufactured, without iteration.

Most of the major components of the OST Concept 2 flight system are shown in Figure 5. A team of systems and discipline engineers at NASA's Goddard Space Flight Center developed this design. 
. Table 1. A comparison of key attributes of OST mission Concept 1 and Concept 2

\begin{tabular}{|c|c|c|}
\hline Parameter & OST Concept 1 & OST Concept 2 \\
\hline Wavelength range $(\mu \mathrm{m})$ & $5-660$ & $5-588$ \\
\hline Telescope Field of View (arcmin) & $25 \times 15$ & $40 \times 15$ \\
\hline Launch Vehicle/Configuration & SLS with $8.4 \mathrm{~m}$ fairing & $\begin{array}{l}\text { Compatible with SLS with } 8.4 \mathrm{~m} \text { fairing; Space X BFR with } \sim 9 \mathrm{~m} \text { fairing; } \\
\text { Blue Origins New Glenn with } \geq 7 \mathrm{~m} \text { fairing }\end{array}$ \\
\hline \multicolumn{3}{|l|}{ Telescope first-order specifications } \\
\hline Aperture size & $\begin{array}{l}9.1 \text { m hexagonal, tip-to-tip; segmented; folded when stowed for launch, } \\
\text { and deployed in space }\end{array}$ & $5.9 \mathrm{~m}$ diameter (circular); no deployment \\
\hline Collecting area $\left(\mathrm{m}^{2}\right)$ & 52 & 25 \\
\hline$f$-number & $f / 12.8$ & $f / 14.0$ \\
\hline Effective Focal Length ( $\mathrm{m}$ ) & 116 & 82.6 \\
\hline Design form & Three-mirror anastigmat, unobstructed (off-axis pupil) & Three mirror anastigmat, on-axis pupil, $0.9 \mathrm{~m}$ central obstruction \\
\hline Operating temperature $(\mathrm{K})$ & 4 & 4 \\
\hline Spatial resolution & $\begin{array}{l}\text { Diffraction limited at } \lambda=30 \mu \mathrm{m} \text { (MISC instrument diffraction limited at } 5 \\
\mu \mathrm{m} \text { with deformable mirror) }\end{array}$ & $\begin{array}{l}\text { Diffraction limited at } \lambda=30 \mu \mathrm{m} \text { (MISC instrument diffraction limited at } 5 \\
\mu \mathrm{m} \text { with deformable mirror) }\end{array}$ \\
\hline Pointing requirements & $\begin{array}{l}\text { Knowledge: } 30 \text { mas (MRSS inertial point); Control: } 44 \text { mas; Jitter: } 22 \text { mas } \\
\text { RMS (at MISC; telescope rqmt TBD) }\end{array}$ & TBD; approximately the same as Concept 1 \\
\hline \multicolumn{3}{|l|}{ Instrument suite } \\
\hline & $\begin{array}{l}\text { Mid-Infrared Imager Spectrometer Coronagraph (MISC) } \\
\text { - Wavelength range: } 5 \text { - } 38 \mu \mathrm{m} \\
\text { - Imaging, spectroscopy } \\
\text { - Transit Spectrometer (<10 ppm stability on a timescale of hours to days) } \\
\text { - Study partners: JAXA, NASA ARC }\end{array}$ & $\begin{array}{l}\text { Mid-Infrared Imager Spectrometer Coronagraph (MISC) } \\
\text { - Wavelength range: } 5 \text { - } 38 \mu \mathrm{m} \\
\text { - Imaging, spectroscopy } \\
\text { - Transit Spectrometer ( } 5 \mathrm{ppm} \text { stability, with a goal of } 1 \mathrm{ppm} \text {, on a } \\
\text { timescale of hours to days) } \\
\text { - Study partners: JAXA, NASA ARC }\end{array}$ \\
\hline & $\begin{array}{l}\text { Medium Resolution Survey Spectrograph (MRSS) } \\
\text { - Wavelength range: } 30 \text { - } 660 \mu \mathrm{m} \\
\text { - Multi-band spectroscopy } \\
\text { - Study partner: JPL }\end{array}$ & \multirow[t]{2}{*}{$\begin{array}{l}\text { Origins Survey Spectrometer (OSS) } \\
\text { - Wavelength range: } 25 \text { - } 588 \mu \mathrm{m} \\
\text { - Multi-band slit spectroscopy, with all bands in one slit; 100 diffraction- } \\
\text { limited beams per slit } \\
\text { - FTS mode provides } \mathrm{R}=43,000 \times(112 \mu \mathrm{m} / \lambda) \\
\text { - High-resolution mode provides } \mathrm{R}=325,000 \times(112 \mu \mathrm{m} / \lambda) \\
\text { - FTS and High-res modes in single diffraction-limited beam } \\
\text { - Study partners: JPL, NASA GSFC }\end{array}$} \\
\hline & $\begin{array}{l}\text { High Resolution Spectrometer (HRS) } \\
\text { - Wavelength range: } 25 \text { - } 200 \mu \mathrm{m} \\
\text { - High-resolution, high-sensitivity spectroscopy } \\
\text { - Study partner: NASA GSFC }\end{array}$ & \\
\hline & $\begin{array}{l}\text { Far-infrared Imager and Polarimeter (FIP) } \\
\text { - Wavelength bands: } 40,80,120,240 \mu \mathrm{m} \\
\text { - Broadband imaging } \\
\text { - } \text { - Different of view } 2.5^{\prime} \times 5^{\prime}, 7.5^{\prime} \times 15^{\prime} \\
\text { - Study partner: NASA GSFC } \\
\end{array}$ & $\begin{array}{l}\text { Far-infrared Imager and Polarimeter (FIP) } \\
\text { - Wavelength bands: } 40,80,120,240 \mu \mathrm{m} \\
\text { - Broadband imaging } \\
\text { - Field of view } 13.5^{\prime} \times 9^{\prime} @ 120 \text { and } 240 \mu \mathrm{m}, 4.5^{\prime} \times 3^{\prime} @ 40 \text { and } 80 \mu \mathrm{m} \\
\text { - Polarization sensitivity: } 0.1 \% \text { in linear and circular; } \pm 1^{\circ} \text { in pol. Angle } \\
\text { - Study partner: NASA GSFC }\end{array}$ \\
\hline & $\begin{array}{l}\text { Heterodyne Receiver for OST (HERO) } \\
\text { - Wavelength bands: } 63-66,111 \text { - } 641 \mu \mathrm{m} \\
\text { - Multi-beam high-resolution spectroscopy } \\
\text { - Study partner: European consortium }\end{array}$ & $\begin{array}{l}\text { Heterodyne Receiver for OST (HERO) } \\
\text { - Wavelength bands: } 617 \text { - } 397 \mu \mathrm{m} ; 397 \text { - } 252 \mu \mathrm{m} ; 252 \text { - } 168 \mu \mathrm{m} \text {; and } 168 \text { - } \\
111 \mu \mathrm{m} \\
\text { - } \mathrm{R}=10^{5}-10^{7} \text { spectroscopy } \\
\text { - Instantaneous FoV: } 2.1^{\prime} \text { x } 2.1^{\prime} @ 480 \mu \mathrm{m} ; 1.3^{\prime} \times 1.3^{\prime} @ 300 \mu \mathrm{m} ; 0.8^{\prime} \times 0.8^{\prime} @ \\
200 \mu \mathrm{m} ; 0.6^{\prime} \times 0.6^{\prime} @ 130 \mu \mathrm{m} \\
\text { - Study partner: European consortium }\end{array}$ \\
\hline
\end{tabular}



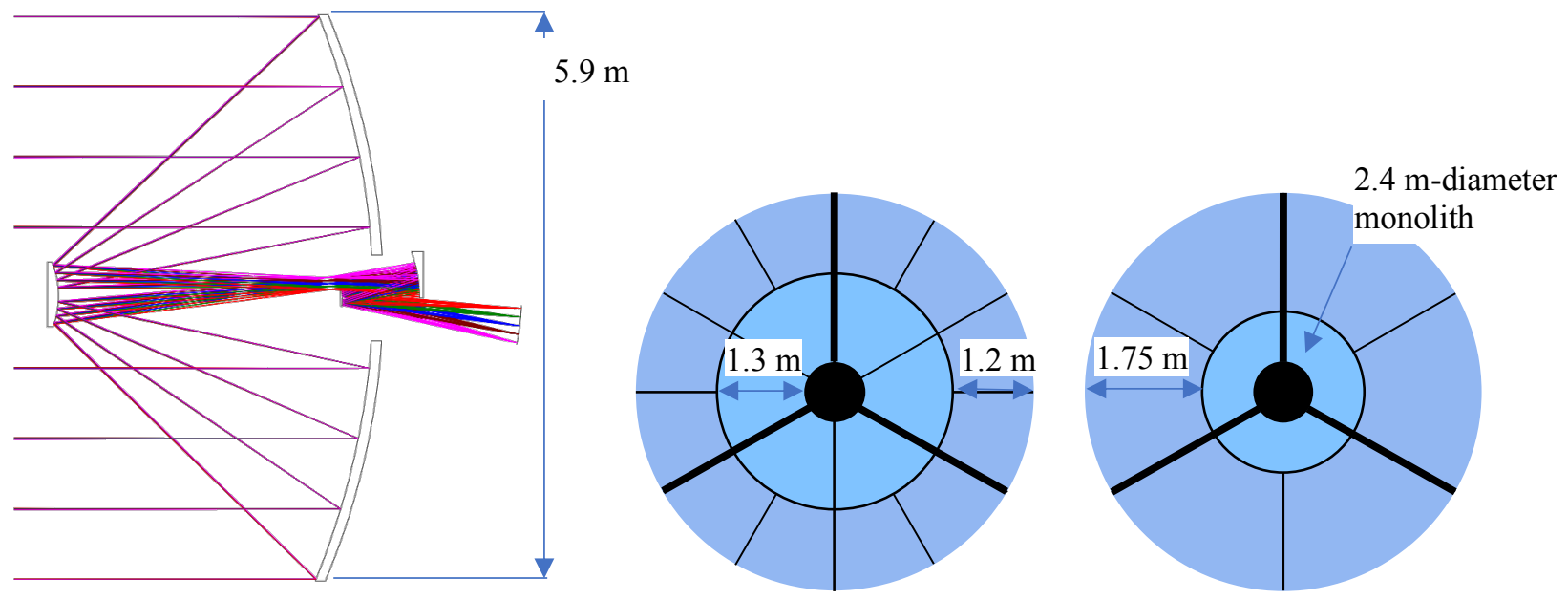

Figure 4. The OST Concept 2 telescope is f/14.0 with a f/0.63 primary mirror. The primary is round, $5.9 \mathrm{~m}$ in diameter, and has a central $0.9 \mathrm{~m}$-diameter hole. In consultation with its industry partners, the study team evaluated four alternative primary mirror segmentation schemes, two of which - shown here - are are still under consideration.

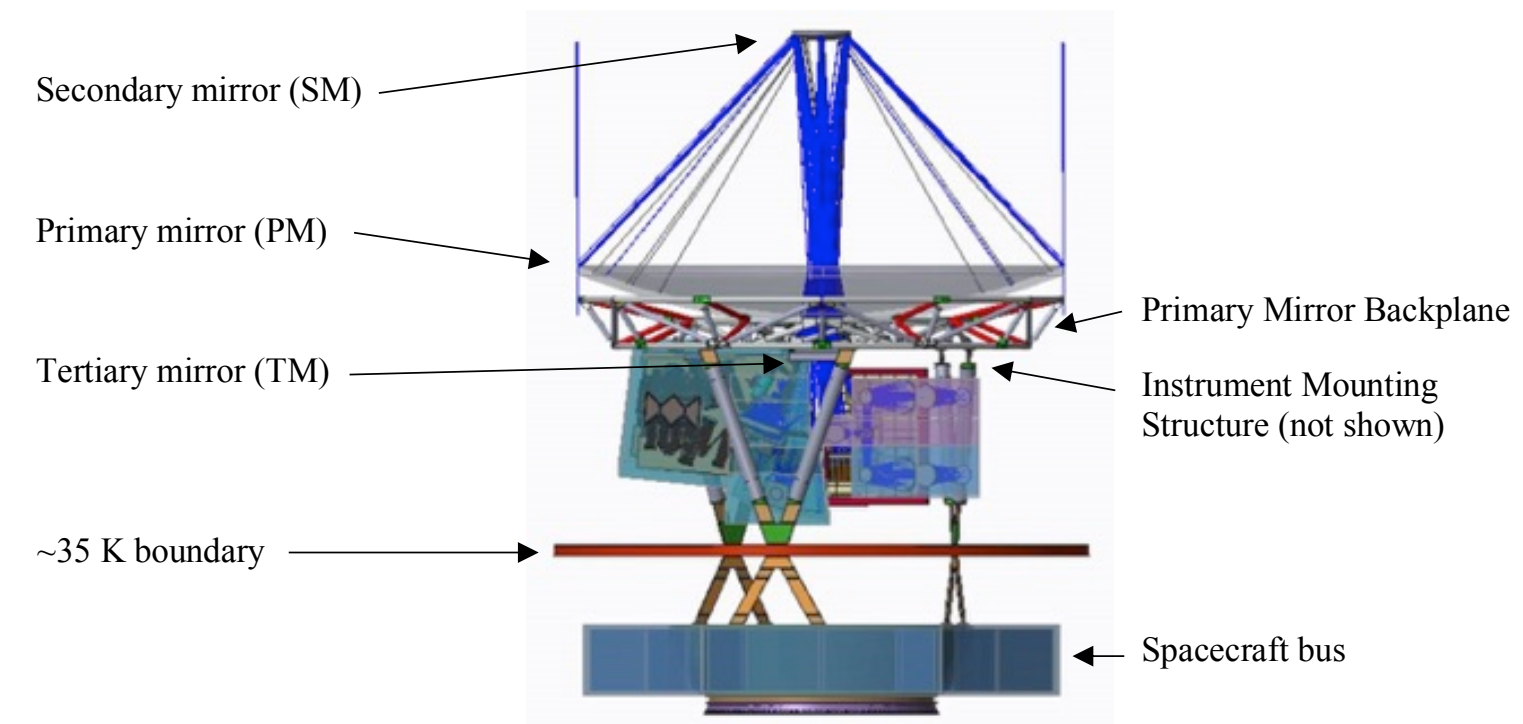

Figure 5. The OST Concept 2 flight system, minus the sunshade and cryocooled barrel and baffle.

\section{OST SCIENCE INSTRUMENTS}

OST's instrument suite is the product of an international team effort involving three NASA Centers. A team led by Prof. Itsuki Sakon in Japan, collaborating with Dr. Tom Roellig at NASA's Ames Research Center, developed a concept for the mid-IR instrument MISC. A European consortium led by Dr. Martina Wiedner at CNES designed the far-IR heterodyne spectrometer HERO. Dr. C. Matt Bradford of Caltech/Jet Propulsion Laboratory designed the far-IR workhorse spectrometer OSS, which inherited a module conceived by Dr. S. Harvey Moseley at NASA's Goddard Space 
Flight Center. Dr. Johannes Staguhn of Johns Hopkins University and Goddard, designed the Far-IR Imager and Polarimeter, FIP. Both OSS and FIP were subjects of week-long studies in Goddard's Instrument Design Lab (IDL). The IDL added design details and developed MELs for the instruments, which serve as foundations for cost models.

For Concept 1, the OST team studied five instruments in sufficient detail to assess their viability, expected performance, capability to satisfy a wide range of science objectives, technical challenges, technology readiness, and cost. For Concept 2, the instrument concepts were descoped to satisfy the prioritized science goals, refined, and reduced in mass, size, and power consumption. Features from the two Concept 1 far-IR direct detection spectrometers - MRSS and HRS - were merged into a single Concept 2 instrument, OSS (Table 1). The Concept 2 instruments are described in companion papers by Sakon et al. (MISC), Bradford et al. (OSS), Staguhn et al. (FIP), and Wiedner et al. (HERO).

All four of the OST instruments are accommodated in the overall volume allocated to instruments in the Concept 2 design. Figure 6 shows ray traces leading to three of the four instruments, MISC, FIP, and HERO. The Concept 2 optical system design allocates space to each of the four science instruments in the telescope's focal plane, as shown in Figure 7.

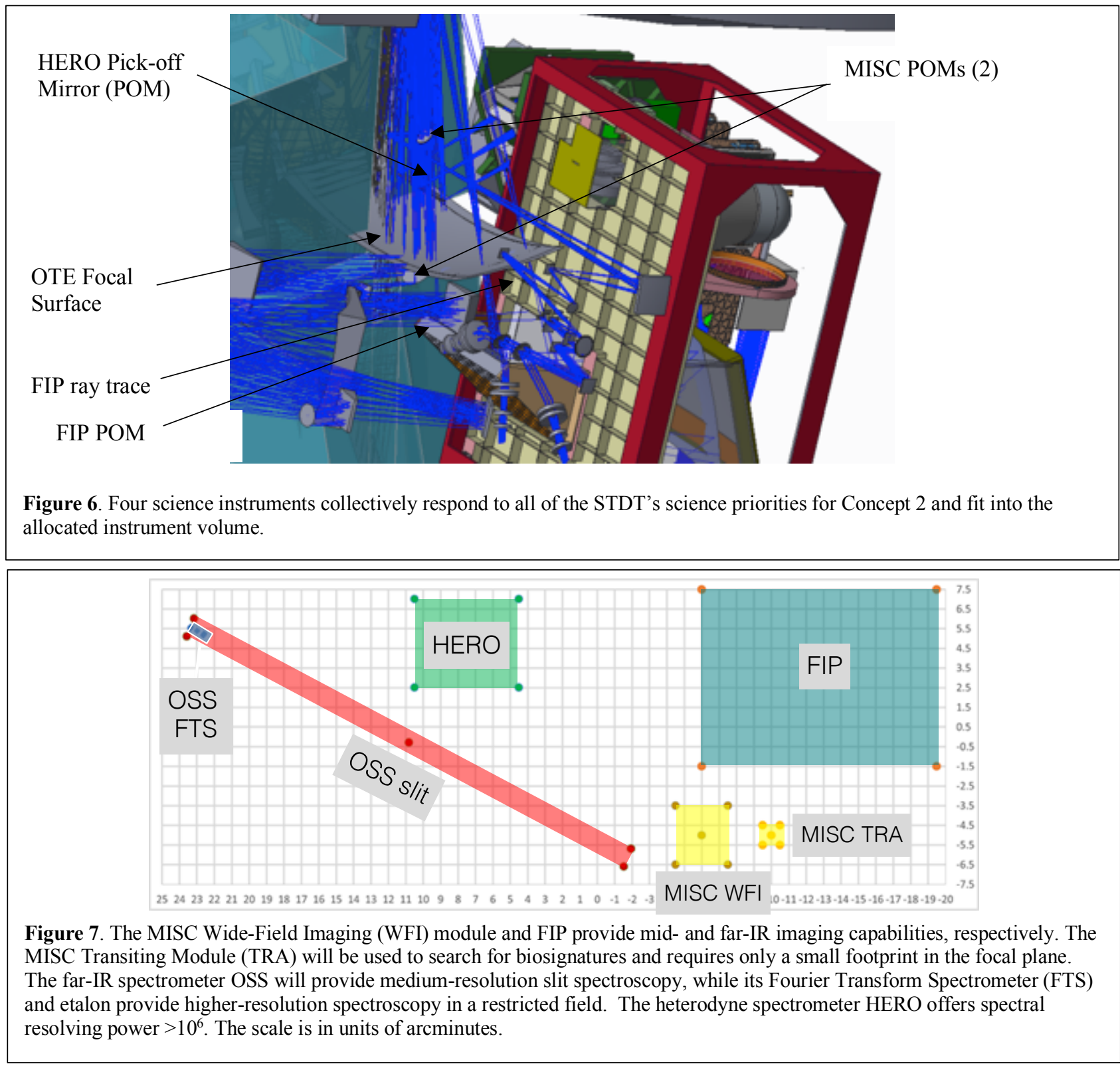


Figure 8 shows the spectral resolving power of each of the four instruments in Concept 2. The estimated spectroscopic sensitivity is shown in Figure 9.

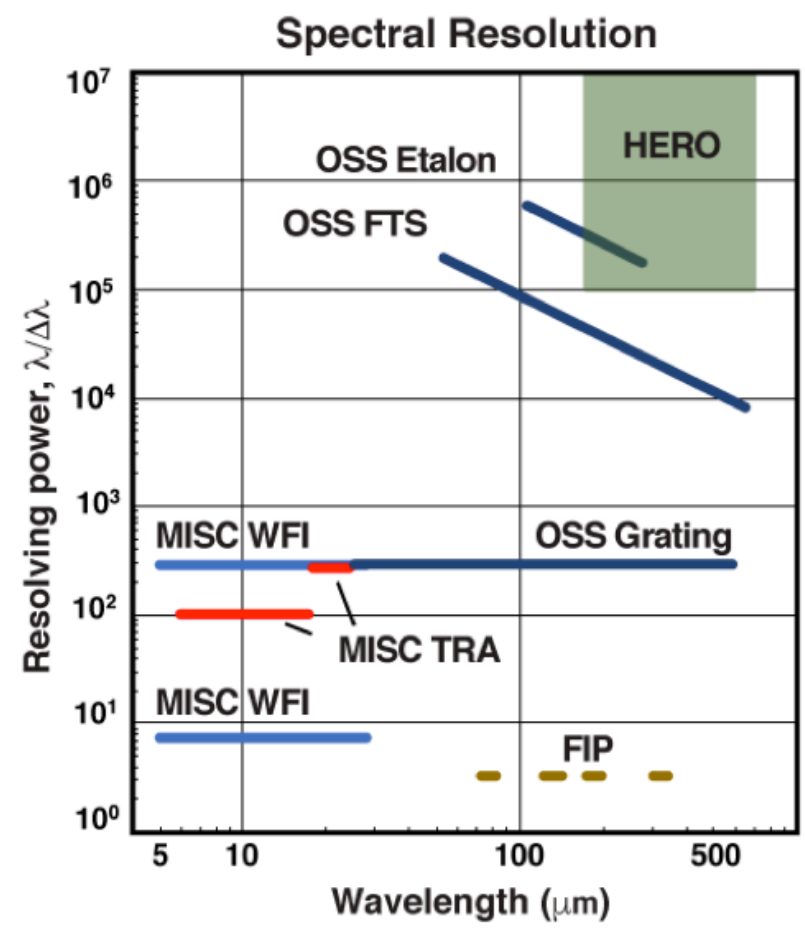

Figure 8. The OST Concept 2 insrrument suite provides a wide range of spectral resolving power at mid- and far-IR wavelengths, enabling measurements that will answer the questions posed at the beginning of section 3 .

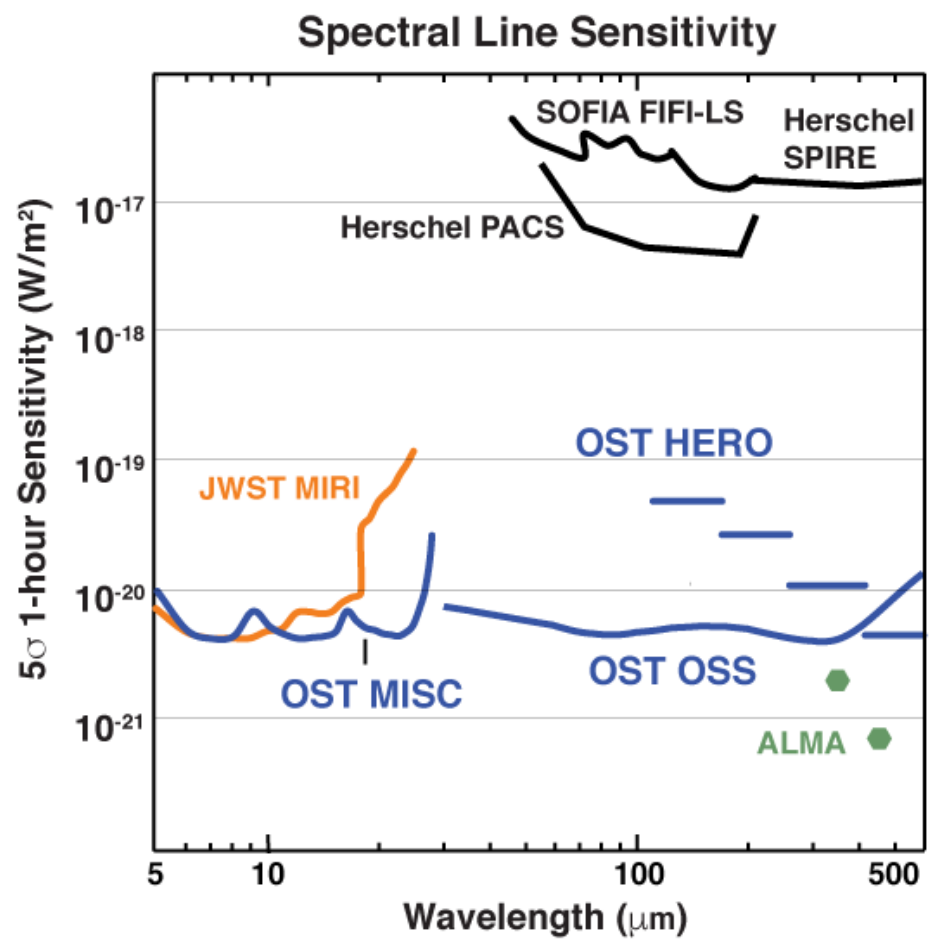

Figure 9. The OST spectrometers offer a two- to three-order of magnitude gain in sensitivity relative to the Herschel instruments PACS and SPIRE, and relative to the FIFI-LS instrument on SOFIA. This improvement is attributable to OST's cold temperature, the lower detector noise in its instruments, and to its larger collecting area. 


\section{MISSION-ENABLING TECHNOLOGY}

Detectors are the most important enabling technology for OST. At far-IR wavelengths, reaching the fundamental sensitivity limits set by astrophysical foregrounds requires use of a cold space telescope and sensitive detectors. The detector sensitivity needed to reach these limits, expressed as the Noise Equivalent Power (NEP) for imaging is around 3 $\mathrm{x} 10^{-19} \mathrm{~W} \mathrm{~Hz}^{-1 / 2}$, whereas the NEP needed for moderate resolution spectroscopy is $3 \times 10^{-20} \mathrm{~W} \mathrm{~Hz}^{-1 / 2}$. Several direct detector technologies are currently in development. Transition Edge Sensors (TES) are based on the sharp change of resistance of a superconductor at its transition. Microwave Kinetic Inductance Detectors (MKIDs) use the superconductor's free electron population temperature dependence, and Quantum Capacitance Detectors (QCDs) use the Cooper-pair breaking that occurs with absorption of far-IR photons. Each of these technologies have their own opportunities and challenges toward the OST goal of large, scalable arrays ( $10^{4}$ to $10^{5}$ pixels), with sensitivities in NEP of $10^{-19}$ to $10^{-20} \mathrm{~W} \mathrm{~Hz}^{-1 / 2}$.

For OST an enabling goal is to multiplex 1000 pixels per channel without cross-talk or loss of sensitivity. An enhancing goal is 3000 pixels per channel. This can be achieved with a combination of microwave SQUIDs, resonant circuits, parametric amplifiers, and/or High Electron Mobility Transistors (HEMTs). Microwave SQUID multiplexing is being developed by SRON in Europe and by NIST and Stanford University in the US. The requirements for far-IR detectors and X-ray detectors for low temperature multiplexing have much overlap, and OST takes advantage of this synergy. Each HEMT or parametric amplifier is a channel. The HEMT or parametric amplifier is located at $4 \mathrm{~K}$ and provides the first level of amplification of the multiplexed signal that is sent to room temperature. For this reason, optimizing HEMTs for low power dissipation is important. The current state-of-the-art in detector readout technology is to use FPGAs to read out multiple channels. The power required is approximately $120 \mathrm{~W}$ per channel. For OST the enabling goal is to dissipate less than $30 \mathrm{~W}$ per channel using ASICs.

Current state-of-the-art, high-Technology Readiness Level (TRL) heterodyne detectors have a noise equivalent temperature of $<1000 \mathrm{~K}$, exist in up to 16-pixel arrays, and have a frequency range of up to $2.1 \mathrm{THz}$. For OST, the frequency range needs to extend to $2.7 \mathrm{THz}(111 \mu \mathrm{m}$ wavelength), and a 9-pixel array would satisfy Concept 2 requirements. The power dissipated per pixel at low temperature will have to decrease from $1 \mathrm{~mW}$ to less than $0.5 \mathrm{~mW}$.

The mid-IR instrument MISC would use arsenic-doped silicon impurity band conduction (Si:As IBC) detectors, or possibly HgCdTe detectors. To achieve OST's exoplanet detection goals in the mid-IR, a factor of 10 improvement in stability is required over the current state-of-the-art as in the James Webb Space Telescope/Mid Infrared Instrument (JWST/MIRI). The OST study team's JAXA partners are examining techniques to improve this stability at the instrument level for MISC.

For detector cooling to $50 \mathrm{mK}$, adiabatic demagnetization refrigerators (ADRs) are currently the only proven technology, although some work has been funded by ESA to develop a continuously recirculating dilution refrigerator (DR). A single-shot DR was flown on Planck, producing $0.1 \mu \mathrm{W}$ of cooling at $100 \mathrm{mK}$ for about 1.5 years, while a three-stage ADR was used on Hitomi, producing $0.4 \mu \mathrm{W}$ of cooling at $50 \mathrm{mK}$ with an indefinite lifetime. In contrast, a TRL 4 continuous $\mathrm{ADR}$ has demonstrated $6 \mu \mathrm{W}$ of cooling at $50 \mathrm{mK}$ with no life-limiting parts ${ }^{20}$. This technology is currently being advanced toward TRL 6 through NASA Strategic Astrophysics Technology funding. Demonstration of a $10 \mathrm{~K}$ upper stage for this machine, as is planned, would enable coupling to a higher temperature cryocooler, such as that of Creare, which has a near-zero vibration technology. The flight control electronics for this ADR are based on the flightproven Hitomi ADR control, which has already achieved TRL 6.

To achieve temperatures lower than about $30 \mathrm{~K}$, OST will use mechanical cryocoolers. Three cryocoolers for $6 \mathrm{~K}$ cooling were developed through NASA's Advanced Cryocooler Technology Development Program. The state-of-the-art for mechanical coolers is represented by Planck, JWST/MIRI, and Hitomi (ASTRO-H). Coolers that can achieve $4.0 \mathrm{~K}$ are considered to be at TRL 4-5, having been demonstrated as a system in a laboratory environment, or as a variant of a cooler that has a high TRL (JWST/MIRI). Mechanical cryocoolers for higher temperatures have already demonstrated impressive on-orbit reliability. The moving components of a $4 \mathrm{~K}$ cooler are similar (expanders) or are exactly the same (compressors) as those that have flown. Further development of these coolers to maximize cooling power per input power for small cooling loads $(<100 \mathrm{~mW}$ at $4 \mathrm{~K})$ and lower mass is desired. There is also a need to minimize the exported vibration from the cooler system. The miniature reverse-brayton cryocoolers in development by Creare are examples of reliable coolers with negligible exported vibration. These coolers are at TRL 6 for $80 \mathrm{~K}$ and TRL 4 for $10 \mathrm{~K}$ operation. 


\section{SUMMARY}

The OST mission concept study team plans to deliver to the 2020 Astrophysics Decadal Survey Committee a scientifically compelling, executable mission concept in June 2019. While still in flux at this time, the study team will recommend a version of OST that likely resembles Concept 2, as presented here. With handoffs from ongoing detector and cryocooler technology maturation efforts and a roadmap for further maturation, all technology needed for OST will reach TRL 5 by 2025, and the mission could be developed for launch in 2036.

\section{REFERENCES}

[1] Pilbratt, G.L., Riedinger, J.R., Passvogel, T., et al, "Herschel Space Observatory. An ESA facility for far-infrared and submilletre astronomy," Astronomy and Astrophysics, 518, L1-L6 (2010)

[2] Neugebauer, G., Habing, H. J., van Duinen, R. et al. "The Infrared Astronomical Satellite (IRAS) mission," Astrophysical Journal Letters, 278, L1-L6 (1984)

[3] Boggess, N.W., et al., "The COBE mission - Its design and performance two years after launch," ApJ, 397, 420 (1992)

[4] Kessler, M.F., Steinz, J.A., Anderegg, M.E. et al., "The Infrared Space Observatory (ISO) mission,” Astronomy and Astrophysics, 315, L27-L31 (1996)

[5] Werner, M.W., Roellig, T.L., Low, F.J., Rieke, G.H. et al., “The Spitzer Space Telescope Mission,” Astrophysical Journal Supplement, 154, 1-9 (2004)

[6] Murakami, H. et al., "The Infrared Astronomical Mission AKARI,” Publ. Astron. Soc. Jap., 59, 369 (2007)

[7] Meixner, M., et al., "Overview of the Origins Space Telescope: science drivers to observatory requirements," paper [10698-22], this volume (2018)

[8] DiPirro, M.J., et al., “The Origins Space Telescope cryogenic-thermal architecture,” paper [10698-44], this volume (2018)

[9] Knight, J.S., et al., "OST wavefront error budget flowdown,” paper [10698-199], this volume (2018)

[10] Lightsey, P.A., et al., "Stray Light overview for the Origins Space Telescope," paper [10698-201], this volume (2018)

[11] Matsuo, T., Satoshi, I., Shibai, H., Takahiro, S., and Yamamuro, T. "A New Concept for Spectrophotometry of Exoplanets with Space-borne Telescopes,” ApJ, 823, 139 (2016)

[12] Bradford, C.M., et al., "The Origins Survey Spectrometer (OSS): a far-IR discovery machine for the Origins Space Telescope," paper [10698-43], this volume (2018)

[13] Sakon, I., et al., "The mid-infrared imager/spectrometer/coronagraph instrument (MISC) for the Origins Space Telescope," paper [10698-42], this volume (2018)

[14] Wiedner, M., et al., "HERO: heterodyne receiver for the Origins Space Telescope," paper [10698-46], this volume (2018)

[15] Staguhn, J., et al., "Origins Space Telescope: the far infrared imager and polarimeter FIP,” paper [10698-45], this volume (2018)

[16] Arenberg, J., et al., "Thermal considerations and architecture for Origins Space Telescope," paper [10698-41], this volume (2018)

[17] “Enduring Quests, Daring Visions: NASA Astrophysics in the Next Three Decades," NASA 2013 Science Roadmap Team (Kouveliotou, C., Chair), https://science.nasa.gov/astrophysics/documents. 
[18] DiPietro, D.A., “A Systems Engineering Approach to Architecture Development," INCOSE International Symposium, 25, 619 (2015)

[19] Kaltenegger, L., "How to Characterize Habitable Worlds and Signs of Life," ARA\&A, 55, 433 (2017)

[20] Shirron, P.J., Canavan E.R., DiPirro M.J., Jackson M., King T.T., Panek J.S., and Tuttle J.G., "A Compact, HighPerformance Continuous Magnetic Refrigerator for Space Missions ," Cryogenics 41, 789 (2002) 